\title{
Quand innovation de service rime avec stratégie de rupture : vers une proposition renouvelée de valeur en B to $B$.
}

\author{
Patricia Coutelle - Brillet \\ Maître de Conférences \\ IAE de Tours / CERMAT \\ patricia.coutelle@univ-tours.fr
}

\author{
Véronique des Garets \\ Professeur des Universités \\ IAE de Tours / CERMAT \\ desgarets@univ-tours.fr
}

\author{
Arnaud Rivière \\ Maître de Conférences \\ IAE de Tours / CERMAT \\ arnaud.riviere@univ-tours.fr
}

Université de Tours - Faculté de Droit, d'Economie et des Sciences Sociales

Quartier des Deux Lions - 50, avenue Jean Portalis - BP 0607 - 37206 Tours cedex 03

\begin{abstract}
Résumé :
Face à une consommation accrue de services industriels, l'innovation de service peut représenter un levier de croissance pour les organisations. Toutefois, dans un environnement fortement compétitif et instable, il est important de s'interroger sur la capacité de ces innovations de services à engendrer une rupture sur les marchés $B$ to $B$. Pour cela, la pertinence d'une proposition alternative de valeur est examinée. Une étude qualitative est réalisée afin d'analyser la diversité des sources de valorisation d'un nouveau service inter-organisationnel. Au-delà des valeurs économique et utilitaire attendues, d'autres types de valeur ont pu être identifiés. Ces résultats conduisent à proposer un cadre d'analyse de la valeur perçue d'un nouveau service dans un contexte B to B qui distingue, selon les catégories d'acteurs concernés par l'innovation, différentes facettes de la valeur perçue, sources potentielles de rupture. Les conséquences d'une telle proposition renouvelée de valeur sont, dans le même temps, appréciées au regard de la chaîne de valeur de l'entreprise.
\end{abstract}

\section{Abstract:}

Facing the increased consumption of industrial services, service innovation can be an appropriate answer for the firms. However, in a highly competitive and volatile environment, it is important to consider the ability of these services innovations to create a rupturing strategy for B to B market. Therefore the relevance of an alternative value proposal is examined. A qualitative study was carried out to analyze the different sources of value for a new inter-organizational service. Beyond the expected economic and utilitarian values, other forms of value were identified. These results lead to propose a framework to analyze the perceived value of a new service in a B to B context which distinguishes, according to people concerned by the innovation, various facets of the perceived value and so, various potential sources of rupture. At the same time, the consequences of such a renewed value proposal are evaluated on the value chain of the company. 


\section{Introduction}

De nombreux travaux ont montré la place croissante prise par les services dans la société (de Brentani, 1995 ; de Brentani, Ragot, 1996; Lovelock et al., 2004). Economistes et gestionnaires se rejoignent pour souligner les mutations auxquelles nous avons pu assister depuis plusieurs décennies. Les premiers parlent de tertiarisation de l'économie, soulignent l'impact des services sur l'emploi, et montrent le développement des besoins en services des entreprises. Les praticiens et les chercheurs en gestion mettent en avant l'importance des services, aussi bien pour les fournisseurs (facteur de différenciation) que pour les clients (meilleure satisfaction d'un besoin). Certains auteurs vont même plus loin en estimant que tout est service (Vargo, Lusch, 2004).

Bon nombre d'organisations de services sont actuellement confrontées à un double défi : affronter une concurrence toujours plus intense et instable sur leur secteur d'activité, et profiter d'une croissance accrue de la consommation de services, en particulier sur les marchés Business to Business ( $B$ to $B$ ).

Pour faire face aux contraintes relatives à l'environnement, la littérature en management souligne le rôle des stratégies de rupture dans le renouvèlement des règles du jeu concurrentiel (Lehmann-Ortega, Roy, 2009 ; Métais et al., 2009).

Concernant les opportunités liées à une demande accrue de services en B to B, les recherches en marketing mettent en évidence le rôle de l'innovation de service comme source de croissance. Bien que les services aient retenu l'attention de bon nombre de chercheurs ces dernières années, l'innovation de service reste encore un sujet sous-exploré, notamment en comparaison avec la thématique des nouveaux produits (Droege et al., 2009). 
Par ailleurs, la majorité des travaux en lien avec ce sujet se situent en Business to Consumer (B to C), mais peu concernent l'innovation de service dans le domaine du B to B. Or celui-ci recouvre des situations variées et présente des spécificités reconnues.

Dans ce contexte, cette recherche s'intéresse ainsi à l'innovation de service, et plus particulièrement à sa capacité à constituer une réelle stratégie de rupture. Une telle appréciation peut notamment s'effectuer par la mobilisation de l'approche de la valeur perçue. En effet, les stratégies de rupture nécessitent de proposer une nouvelle valeur pour les clients, et ne peuvent être qualifiées de succès que si les acheteurs adhèrent à la proposition qui leur est faite (Lehmann-Ortega, Roy, 2009). Le terme de rupture n'est toutefois réservé qu'au seul cas où une modification de la chaîne de valeur peut être constaté (Lehmann-Ortega et Schoettl, 2005). Dans ce cadre, il est donc utile de s'interroger sur la multiplicité potentielle des sources de valorisation d'une innovation de service en B to B. Cette réflexion permettra d'évaluer la pertinence, pour les entreprises, de proposer à leurs clients industriels une valeur autre qu'utilitaire ou économique, traditionnellement offerte à leurs acheteurs. Par ailleurs, les conséquences d'une telle modification de valeur, au sein de l'organisation, seront examinées.

Après avoir défini le cadre théorique de cette recherche articulé autour des notions d'innovation de service, de stratégie de rupture et de valeur perçue, la méthodologie de l'étude réalisée est présentée. Puis, les résultats obtenus sont analysés, permettant d'identifier des sources de rupture potentielles dans le cadre des innovations de services en B to B. Enfin, les conclusions de l'étude sont discutées, les apports et limites de ce travail sont précisés, et plusieurs pistes de réflexion futures sont suggérées. 


\section{D’une innovation de service à une stratégie de rupture : une analyse sous le prisme de la valeur perçue}

Après avoir présenté l'enjeu des innovations de services inter-organisationnels, leur capacité à modifier les règles du jeu concurrentiel est examinée au travers de l'étude des conditions de mise en œuvre des stratégies de rupture. Dans ce cadre, l'importance d'une prise en compte de la valeur perçue, dans l'appréhension d'une nouvelle offre en $B$ to $B$, est soulignée.

\subsection{Service et innovation de service dans un contexte Business to Business}

Les services représentent aujourd'hui une part prépondérante de l'activité économique dans les pays développés où ils sont en constante croissance. Ce constat se traduit notamment, sur les marchés $B$ to $B$, par une demande accrue de prestations par les entreprises industrielles et par l'essor du nombre d'acteurs opérant dans le secteur tertiaire. L'attrait renforcé des services professionnels ${ }^{1}$ pour les acheteurs a plusieurs causes, issues de l'entreprise elle-même ou imposées par l'environnement :

- la complexité croissante des biens industriels rend plus que jamais nécessaire la consommation de services " supports » aux produits (services avant et après vente : conseil-expertise, formation, maintenance...). D'ailleurs, on parle souvent, en B to B, de "vente de systèmes " pour décrire cette combinaison de produits et services (Azimont et al., 1999).

\footnotetext{
1 Les termes "services professionnels", "services inter-organisationnels" ou bien encore "services industriels » désignent, dans cet article, les services offerts sur les marchés business to business.
} 
- le besoin de certaines entreprises de se recentrer sur leurs savoir-faire fondamentaux peut les conduire à externaliser une partie de leurs activités ne se situant pas au cœur de leurs compétences (exemple de la logistique) (Paché et des Garets, 1997).

- la croissance de la production industrielle entraîne une consommation accrue de services par les entreprises (transport, publicité, traitement de l'information...).

- l'apparition récente de nouveaux services et la nécessité de s'adapter aux nouveaux impératifs du marché stimulent la demande des entreprises (places de marchés électroniques, e-procurement, e-services...).

- l'internationalisation des échanges renforce la compétition entre les organisations et incite de nombreux décideurs à rechercher de nouvelles sources de différenciation : l'achat de services peut conduire à acquérir un avantage concurrentiel via un enrichissement de l'offre commercialisée (Rivière, 2009).

Dans ce contexte fortement marqué par la consommation de services interorganisationnels, l'innovation de service constitue une source de croissance potentielle pour les fournisseurs (de Brentani, 1995). L'innovation de service peut être de nature très différente, avec des degrés d'innovation variés (Gallouj, Weinstein, 1997 ; Gallouj, Savona, 2009). Par exemple, Phan (2007) distingue quatre catégories d'innovations: les services créés en adaptant ou modifiant un service existant, les services nouveaux pour l'entreprise mais déjà présents sur le marché, les services déjà existants mais adaptés pour un nouveau marché (ou segment), et enfin les services totalement nouveaux. Dans le cadre spécifique des marchés $B$ to $B$, le processus d'adoption d'un nouveau service inter-organisationnel est confronté à plusieurs spécificités : 
- L'adoption d'une nouveauté est issue d'une décision collective prise par l'ensemble des membres du centre d'achat (entité informelle) de l'entreprise : utilisateurs potentiels du service, décideurs en charge de la décision finale, prescripteurs de l'achat, et acheteurs (du service achat) (Malaval, Bénaroya, 2009).

- Le Nagard et Manceau (2005) distinguent la décision d'une entreprise d'adopter une nouvelle offre et l'adoption de l'innovation par les salariés (adoption intraorganisationnelle).

- Fréquemment, les fournisseurs sont confrontés à l'existence d'une demande dérivée qui nécessite de ne pas prendre uniquement en compte les besoins des clients directs, mais également ceux des clients des clients (Pinard-Legry et al., 1996).

L'instabilité permanente du contexte économique, couplée à la compétition croissante entre les firmes, incitent ces dernières à rechercher, par leurs innovations, la création de réelles ruptures sur le marché. L'objectif est ainsi de démontrer leur capacité à revisiter les règles du jeu au sein de leurs industries. Plus précisément, la création d’une rupture peut entraîner, à la fois, une déstabilisation de l'environnement concurrentiel et une modification des rapports de domination. Toutefois, plusieurs conditions doivent êtres remplies avant de considérer l'innovation de service comme une réelle stratégie de rupture.

\subsection{Les conditions de mise en œuvre d'une stratégie de rupture}

Selon Lehmann-Ortega et Roy (2009), " une stratégie de rupture consiste pour une entreprise à revisiter de manière radicale les règles du jeu concurrentiel en proposant une nouvelle valeur au client en vue de créer ou d'étendre un marché à son avantage ». Cette définition met clairement, au cœur des stratégies de rupture, la notion de " valeur client ». 
En vue d'initier une rupture sur un marché, Métais et al. (2009) proposent quatre leviers d'actions :

- questionner les forces et faiblesses des acteurs de la concurrence,

- modifier les facteurs clés de succès,

- remettre en cause les cadres de référence de l'industrie,

- questionner les frontières et les marges : la rupture peut naître du rapprochement inattendu de deux rationalités éloignées. Par exemple, il est fréquent de distinguer, au sein d'une offre, une dominante « émotionnelle » ou " fonctionnelle ». Le passage de l'une à l'autre peut ainsi être source de rupture.

En B to B, les entreprises mettent traditionnellement en avant la performance ou l'avantage économique de leur offre. Nous pouvons alors nous interroger sur la pertinence d'une proposition différente de valeur, davantage axée sur des dimensions non-utilitaires.

Pour être avérée, la rupture doit aboutir, au final, à une modification de la chaîne de valeur. Ce mouvement stratégique concernant à la fois l'offre telle qu'elle est perçue par le consommateur, et le processus de construction de l'offre, permet de distinguer clairement les stratégies de rupture des stratégies perturbatrices ou incrémentales (Lehmann-Ortega et Schoettl, 2005 ; Asselineau et Pire-Lechalard, 2009).

\subsection{La valeur perçue d'une innovation de service en Business to Business}

Traditionnellement, la valeur perçue est abordée en marketing selon une vision dichotomique, articulée autour des notions de valeur d'achat et de valeur de consommation. 
La valeur d'achat s'inscrit dans une perspective rationnelle et cognitive. Elle résulte d'une confrontation entre les bénéfices et les sacrifices associés à l'achat d'un produit. II s'agit d'une évaluation pré-achat (Zeithaml, 1988).

La valeur de consommation est une préférence relative, née de l'interaction entre un consommateur et une offre. Elle résulte de l'expérience de consommation ou de possession d'un produit ou service, et présente un caractère multidimensionnel. Elle a surtout été mobilisée dans la sphère des services (expériences culturelles, distribution...) (Filser, Plichon, 2004 ; Mencarelli, 2008). Holbrook (1994, 1999, 2006), dont les travaux constituent un apport majeur, a proposé une typologie de la valeur de consommation. Elle est principalement articulée autour de deux dimensions clés et permet de répertorier les différents types de valeur potentiels au sein d'une expérience de consommation² :

- Valeur extrinsèque / intrinsèque : la valeur d'un produit est extrinsèque (le produit est un moyen pour atteindre des fins qui lui sont extérieures) ou intrinsèque (l'expérience de consommation associée au produit, ou la possession de l'objet, est recherchée et appréciée en tant que telle).

- Valeur orientée vers soi / valeur orientée vers les autres : la valeur est orientée vers soi (fonction de son intérêt personnel) ou orientée vers les autres (la famille, les amis, les voisins, la société, le monde...). Dans ce dernier cas, le consommateur juge les objets et les expériences en fonction des autres et pour les autres.

Sur la base de ces deux critères (valeur extrinsèque / intrinsèque et valeur orientée vers soi / vers les autres), et à partir des travaux d'Holbrook (2006), il est possible d'identifier cinq formes principales de valeur (tableau 1).

\footnotetext{
${ }^{2}$ Une troisième dimension (valeur active / réactive) a été identifiée à l'origine par Holbrook (1994, 1999). Toutefois, en raison de sa complexité d'appréhension, cette distinction a été peu reprise dans la littérature.
} 
Tableau 1 : Les différentes facettes de la valeur de consommation

\begin{tabular}{|c|c|c|}
\hline & Extrinsèque & Intrinsèque \\
\hline $\begin{array}{c}\text { Orientée } \\
\text { vers soi }\end{array}$ & $\begin{array}{l}\text { Valeur économique } \\
\text { Rapport qualité / prix } \\
\underline{\text { Valeur fonctionnelle }} \\
\text { Provient des fonctionnalités, des } \\
\text { caractéristiques, de la qualité, des } \\
\text { performances, de « l'excellence » du } \\
\text { produit }\end{array}$ & $\begin{array}{l}\text { Valeur émotionnelle } \\
\text { Provient de sensations ou d'états } \\
\text { sentimentaux et affectifs qu'un produit } \\
\text { provoque (jeu, plaisir, beauté, } \\
\text { esthétique) }\end{array}$ \\
\hline $\begin{array}{c}\text { Orientée } \\
\text { vers les } \\
\text { autres }\end{array}$ & $\begin{array}{l}\text { Valeur sociale } \\
\text { Concerne la construction de l'image } \\
\text { de soi qu'un individu renvoie aux } \\
\text { « autres » }\end{array}$ & $\begin{array}{l}\text { Valeur altruiste } \\
\text { Fréquemment abordée au travers de la } \\
\text { valeur éthique qui repose sur la vertu, } \\
\text { la justice ou la morale }\end{array}$ \\
\hline
\end{tabular}

Source : adapté d'Holbrook (2006)

Malgré les préoccupations actuelles des entreprises concernant l'accroissement de la valeur perçue de leur offre, la littérature en marketing B to B présente peu de travaux consacrés à ce sujet (Ulaga, Chacour, 2001). Les études portent généralement sur la nature de la valeur d'une offre inter-organisationnel (Lindgreen, Wynstra, 2005), son caractère dynamique (Flint et al., 2002 ) ou sur le processus de création de valeur (Grönroos, 2011). En marketing B to B, la valeur a principalement été étudiée au travers de la valeur d'achat, dont une des composantes importantes semble résider dans l'interaction client-fournisseur ${ }^{3}$ (valeur relationnelle) (Corsaro, Snehota, 2010). Par ailleurs, peu de travaux se sont intéressés spécifiquement à la valeur perçue d'un service en B to B. Seuls La et al. (2009) ont montré

\footnotetext{
${ }^{3} L^{\prime}$ importance de la relation client / fournisseur en B to B, soulignée en particulier par les travaux s'inscrivant dans le courant interactif de la modélisation du comportement d'achat industriel (voir Cova et Salle, 1992, pour une synthèse), peut notamment s'illustrer au travers de la vente de solutions ou du marketing de projet qui nécessitent tous deux une « intimité avec le client » (voir Azimont et al., 1999).
} 
que le degré de valorisation d'un service inter-organisationnel est fortement influencé par la performance perçue de l'offre.

Afin d'apprécier la capacité des innovations de services à s'inscrire dans une stratégie de rupture, cette présente recherche vise à mieux cerner les différentes sources potentielles de la valorisation d'un nouveau service dans un contexte inter-organisationnel. Pour se faire, l'approche par la valeur de consommation, mieux adaptée au domaine des services, est adoptée $e^{4}$

\section{L'exploration de la nature de la valeur perçue d'une innovation de service}

\section{en $B$ to $B$}

Afin de mieux comprendre la nature de la valorisation d'une innovation de service en $B$ to $B$, une étude qualitative a été réalisée. Après avoir explicité les modalités méthodologiques de l'investigation menée, les principaux résultats obtenus sont exposés.

\subsection{Une méthodologie qualitative}

Treize entretiens semi-directifs ont été conduits en face à face auprès de chefs d'entreprises (dans la majorité des cas) ou de responsables d'achat appartenant à des organisations de secteurs d'activité variés (transport, conseil, immobilier, services à domicile...). Ces personnes interviewées ont été choisies en raison de leur vision globale de l'entreprise et de ses besoins, ainsi que de leur pouvoir de décision (Pinard-Legry et al., 1996). Ces entretiens individuels, d'une durée d'environ 45 minutes, ont été menés en trois temps :

\footnotetext{
4 Le domaine des services est particulièrement associé au vécu d'expériences que seule la valeur de consommation peut pleinement prendre en compte.
} 
- les interviewés ont d'abord décrit leur comportement d'achat de services de manière générale (type de services consommés, critères de choix...).

- les responsables ont ensuite été interrogés sur leur processus d'adoption habituel d'une innovation de service (recherche d'informations, critères de décision, conséquences recherchées...).

Pour ces deux premières étapes, nous avons privilégié un mode d'entretien de type phénoménologique favorisant l'expression et le souvenir d'évènements antérieurs, d'expériences vécues.

- les individus ont enfin été mis en situation de manière à décrire leur processus d'adoption d'une innovation concernant une liste de services proposés (crèche d'entreprise, services de bien-être, e-services...).

Le nombre d'entretiens a été défini selon le principe de saturation sémantique. Les données qualitatives ont été traitées par analyse de contenu thématique (manuelle et informatisée).

\subsection{Résultats}

Deux principaux résultats sont issus de cette étude exploratoire. Le premier distingue différents destinataires de la création de valeur d'une innovation de service. La valeur associée à une nouvelle prestation peut être perçue par l'un des membres du centre d'achat, mais aussi par l'ensemble de l'organisation ou par les clients de l'organisation. Le second résultat fait état de la nature multidimensionnelle de la valeur relative aux innovations de services, soulignant de ce fait la pertinence du choix du cadre inspiré d'Holbrook (1994, 1999, 2006) pour analyser la valeur en B to B. A l'issue des entretiens, plusieurs facettes de la valeur apparaissent : les dimensions économique et fonctionnelle classiquement associées 
à la valeur en $B$ to $B$, mais également des dimensions moins utilitaires, telles que les dimensions sociale ou émotionnelle.

\subsubsection{Les destinataires de la création de valeur des innovations de services en contexte B} to B

Dans le contexte des innovations de services en B to B, il est possible d'identifier des sources de valeur orientée vers l'un des membres du centre d'achat ou vers d'autres acteurs extérieurs au centre d'achat de l'organisation cliente (le reste de l'organisation et ses clients).

Pour les membres du centre d'achat de l'organisation cliente, la perception de valeur apparaît clairement dans certains verbatims :

Ind ${ }^{5} 1$ « On peut avoir deux différentes approches, soit on réfléchit en termes d'apports pour le client ou alors, en termes de position personnelle par rapport à l'innovation ".

Bien qu'extérieurs au centre d'achat, d'autres acteurs appartenant à l'organisation peuvent apprécier la valeur créée. Elle peut se traduire par une meilleure performance perçue de l'entreprise, une amélioration de l'image interne de l'organisation ou bien encore une plus grande reconnaissance des salariés (amélioration du climat de travail) :

Ind 3 "L'innovation pour une entreprise est pour moi synonyme de performance et de productivité... Elle permet également d'améliorer l'image au sein de l'entreprise. Ce dernier point est alors essentiel pour garder un bon climat de travail »

${ }^{5}$ Ind = individu 
Enfin, la valeur d'une innovation de service B to B peut concerner les autres parties prenantes de l'entreprise (fournisseurs, clients, concurrents) :

Ind 5 " II est important de capter l'attention de nos clients par des actions vivantes, qui montrent le dynamisme de l'entreprise, sa capacité d'adaptation »

\subsubsection{Les différentes sources de valeur identifiées}

Les dimensions économique et fonctionnelle de la valeur sont bien présentes dans la plupart des verbatims collectés auprès des responsables interrogés :

Ind $\mathbf{2}$ "Le critère de choix est économique à la base... on est sur une logique purement économique, si j'investis 100, combien ça me rapporte à terme ... II y a la productivité »

Ind 8 « Evidemment le rapport qualité / prix »

La valeur fonctionnelle d'un nouveau service semble trouver son origine dans la possibilité d'améliorer la productivité de l'entreprise, de réaliser un gain de temps significatif, ou bien d'assurer la prise en charge d'activités pour lesquelles l'entreprise n'a pas de compétences spécifiques.

Hormis les dimensions fonctionnelle et économique de la valeur, d'autres dimensions, abordées par Holbrook (1994, 1999, 2006), apparaissent également dans l'analyse : les aspects émotionnel et social de la valeur.

La facette émotionnelle de la valeur se révèle dans le discours des répondants par des verbes tels qu' " aimer » ou des adjectifs tels que « ludique » :

Ind $\mathbf{2}$ "Dans la réalité, il y en a beaucoup qui fonctionnent sur choix personnel parce que j'aime l'innovation, ça me plait. Il y a des vraies postures, il y a aussi des rétifs, mais tu as des gens qui sont fidèles à ce genre de postures par nature » 
Ind 12 «Ce service n'est pas anti-stress mais ludique »

En comparaison avec la valeur émotionnelle, la valeur sociale est beaucoup plus présente dans les verbatims étudiés :

Ind $\mathbf{2}$ " Du coup, on en attend quelque chose à terme, pas forcément quelque chose d'ordre économique, mais plus de l'ordre de l'image de l'entreprise »

Enfin, conformément à la littérature (Corsaro, Snehota, 2010), la valeur perçue par un acteur ne provient pas uniquement de l'interaction avec le service mais aussi avec le fournisseur de service. L'analyse de contenu menée permet de confirmer l'existence et l'importance de cette valeur relationnelle. Elle dépend des qualités ainsi que de l'expertise du personnel en contact :

Ind 10 "Le contact avec le personnel de vente s'est très bien passé, le contact s'est fait par téléphone mais ils ont su bien déterminer mes besoins et répondre à mes questions. La facilité à les joindre était également importante, pas de temps d'attente quand on appelle, toujours les mêmes contacts au téléphone... la relation est très importante... Un rapport de confiance doit se créer entre les deux parties, l'acheteur et le vendeur »

Dans le domaine de l'innovation de service, cette interaction acheteur / vendeur, susceptible d'être importante aux yeux de certains clients industriels, peut conduire à une adaptation particulière du fournisseur.

Ind 6 «L'innovation, ce n'est pas tant avoir l'idée mais c'est accompagné les entreprises à intégrer cette nouvelle idée dans leur entreprise » 
A l'issue de cette analyse sur la nature de la valorisation d'une offre de service en $B$ to $B$, l'existence de différentes sources de valeur, fonctionnelle, économique, mais également émotionnelle, sociale ou bien encore relationnelle, ont donc pu être identifiées.

\section{Discussion, apports et limites}

A l'issue de l'étude menée, un cadre d'analyse de la valeur perçue d'une innovation de service en B to B peut être proposé. Par ailleurs, une telle proposition renouvelée de valeur nécessite d'examiner ses conséquences sur la chaîne de valeur de l'entreprise.

\subsection{Proposition d'un cadre d'analyse de la valeur perçue d'une innovation de service en $B$ to $B$}

Afin d'appréhender la valorisation d'une innovation de service en $B$ to $B$, deux éléments majeurs de cette recherche doivent être rappelés :

- la revue de la littérature a souligné l'enjeu de la valeur de consommation dans l'appréciation de la diversité des sources de valeur d'une offre. La matrice d'Holbrook (1994, 1999, 2006) représente une base de réflexion intéressante. Le travail de structuration des sources de valeur, proposé par cet auteur, constitue un apport théorique essentiel dans la littérature marketing.

- l'étude empirique, menée dans cette recherche, a mis en évidence, à la fois, différents destinataires de la création de valeur et différentes dimensions de la valeur d'une innovation de service.

Ainsi, en s'inspirant des travaux d'Holbrook (1994, 1999, 2006), il est possible de croiser les deux résultats obtenus à l'issue de la phase qualitative : Diversité des destinataires de la 
valeur créée * Diversité des sources de valeur. Cet essai de clarification de la nature de la valeur, dans le cadre d'une innovation de service en B to B, est proposé dans le tableau 2.

Tableau 2 : Proposition d'un cadre d'analyse de la valeur perçue d'une innovation de service en $B$ to $B$

\begin{tabular}{|c|c|c|c|c|}
\hline & \multicolumn{2}{|c|}{ Valeur issue de l'interaction consommateur / service } & \multirow{2}{*}{$\begin{array}{l}\text { Valeur issue de } \\
\text { l'interaction } \\
\text { client / } \\
\text { fournisseur }\end{array}$} \\
\hline & & Valeur extrinsèque & Valeur intrinsèque & \\
\hline \multirow{3}{*}{ 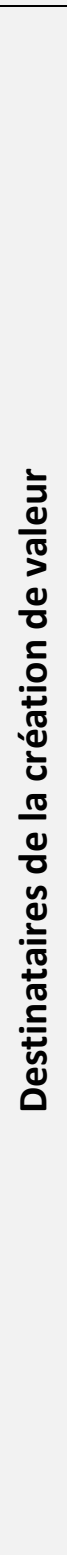 } & $\begin{array}{l}\text { Valeur orientée } \\
\text { vers l'un des } \\
\text { membres du } \\
\text { centre d'achat } \\
\text { de } \\
\text { l'organisation } \\
\text { cliente }\end{array}$ & $\begin{array}{c}\text { Valeur fonctionnelle } \\
\text { (gain de temps dans son propre } \\
\text { travail) } \\
\text { Valeur sociale } \\
\text { (image d'innovateur du } \\
\text { décideur) }\end{array}$ & $\begin{array}{l}\text { Valeur émotionnelle } \\
\text { (plaisir lié au fait de } \\
\text { décider d'adopter } \\
\text { une innovation) }\end{array}$ & $\begin{array}{c}\text { Valeur } \\
\text { relationnelle } \\
\text { (rapport de } \\
\text { confiance avec } \\
\text { le fournisseur) }\end{array}$ \\
\hline & $\begin{array}{l}\text { Valeur orientée } \\
\text { vers l'ensemble } \\
\text { de } \\
\text { l'organisation } \\
\text { cliente }\end{array}$ & $\begin{array}{c}\text { Valeur fonctionnelle } \\
\text { (meilleure productivité, gain de } \\
\text { temps, service mieux assuré par } \\
\text { un prestataire extérieur) } \\
\text { Valeur économique } \\
\text { (contribue à la santé financière } \\
\text { et à la compétitivité de } \\
\text { l'entreprise) } \\
\text { Valeur sociale } \\
\text { (valorisation de l'image de } \\
\text { l'entreprise en interne auprès } \\
\text { des salariés) }\end{array}$ & $\begin{array}{c}\text { Valeur émotionnelle } \\
\text { (meilleure } \\
\text { considération de la } \\
\text { part de } \\
\text { l'encadrement / } \\
\text { direction, sentiment } \\
\text { de reconnaissance } \\
\text { éprouvé par les } \\
\text { salariés, ambiance } \\
\text { conviviale) }\end{array}$ & \\
\hline & $\begin{array}{l}\text { Valeur orientée } \\
\text { vers les clients } \\
\text { de } \\
\text { l'organisation } \\
\text { cliente }\end{array}$ & $\begin{array}{c}\text { Valeur fonctionnelle } \\
\text { (mieux répondre aux besoins et } \\
\text { attentes des clients) } \\
\text { Valeur sociale } \\
\text { (améliorer l'image de } \\
\text { l'entreprise cliente auprès de } \\
\text { ses propres clients : image plus } \\
\text { professionnelle) }\end{array}$ & & \\
\hline
\end{tabular}


Parmi les différentes dimensions de la valeur d'une innovation de service en $B$ to $B$, trois d'entre elles (valeurs fonctionnelle, émotionnelle et sociale) peuvent être perçues par différentes catégories d'acteurs : l'acheteur ou le centre d'achat de l'organisation cliente, l'organisation cliente dans son ensemble (les salariés) mais aussi les propres clients de l'organisation cliente.

Si la plupart des valeurs définies par Holbrook $(1994,1999,2006)$ sont présentes dans notre analyse, on peut remarquer une absence de perception de la valeur éthique. Ceci ne condamne pas pour autant son existence. En effet, certaines innovations de services pourraient tout à fait véhiculer une telle valeur en $B$ to $B$ (choix d'un prestataire respectueux de l'environnement ou relevant de l'économie sociale et solidaire). Des recherches complémentaires sont donc nécessaires afin d'analyser cette proposition.

A ce stade, nous avons donc pu démontrer que l'innovation de service, en cherchant à délivrer aux clients une valeur non-rationnelle (émotionnelle, sociale, relationnelle), peut constituer une voie pertinente pour modifier les règles du jeu concurrentiel. Toutefois, pour l'assimiler à une stratégie de rupture, encore faut-il qu'elle soit en mesure de modifier la chaîne de valeur de l'organisation.

\subsection{Impact du renouvellement de la proposition de valeur sur la chaîne de valeur de l'organisation}

S'intéresser à la modification de la chaîne de valeur revient, finalement, à s'interroger sur la manière de proposer ces nouvelles valeurs évoquées précédemment.

Pour Hamel (1998a, 1998b) et Markides (1997, 1998, 1999), un des facteurs déterminants de l'émergence d'une stratégie de rupture est la culture de l'entreprise. En passant d'une 
logique " produit » (consistant à vendre des performances et un bon rapport qualité / prix) à une logique «valeur » (consistant davantage à vendre des émotions, un statut, du relationnel), la culture des organisations, évoluant en B to $B$, doit être intégralement repensée.

La proposition renouvelée de valeur peut affecter à la fois les activités principales et les activités de soutien de la chaîne de valeur.

En effet, cette évolution dans la nature de la valeur délivrée aux clients nécessite une modification profonde du maillon de la chaîne de valeur "gestion des ressources humaines ", en particulier en matière de compétences requises du personnel du fait de l'importance de l'interaction client / vendeur.

De même, cette modification dans la valeur offerte conduit à repenser totalement le processus de marketing / vente. La variable prix perd de son importance, alors que la communication et la distribution (via notamment la force de vente) sont amenées à revoir intégralement leur discours (argumentation) et leur approche du client.

Enfin, la proposition d'une valeur relationnelle peut entraîner, par son intensité, un renouvellement des pratiques du fournisseur à l'égard de ses clients. Cette évolution dans les rapports acheteurs / vendeurs peut provoquer une réelle modification de la chaîne de valeur du fournisseur, mais aussi de celle du client. L'ampleur de ces modifications dépend principalement du degré de co-création du service.

\subsection{Apports et limites de la recherche}

D'un point de vue théorique, l'influence du cadre d'analyse de la valeur d'Holbrook (1994, 1999, 2006), traditionnellement utilisé en B to C, a permis d'aboutir à un travail de 
structuration des sources de valeur d'une innovation de service en B to B. Par une meilleure appréhension de la nature de la valeur dans un contexte inter-organisationnel, cette contribution permet d'identifier des sources de rupture issues du déplacement de la valeur sur un axe rationnel / non rationnel. Ce résultat suscite plusieurs apports managériaux.

L'identification des différentes sources de valeur représente une aide précieuse lors de la conception de nouvelles prestations. Les innovations de services fournies par les entreprises sont traditionnellement orientées vers des sources de valeur fonctionnelle et économique. Notre analyse montre qu'elles peuvent être associées à des aspects émotionnels, sociaux, relationnels. Le positionnement voulu pour les services proposés peut alors s'avérer très varié et en rupture avec les pratiques traditionnelles.

La mise en exergue de la diversité des sources de valeur engendrée par les services industriels innovants peut déboucher sur l'utilisation de nombreux axes de communication. Là aussi, toutes les sources de valeur peuvent potentiellement être mises en avant, permettre une différenciation auprès des acheteurs et nourrir une argumentation commerciale.

Enfin, nous avons vu que les dimensions de la valeur identifiées peuvent s'adresser non seulement aux membres du centre d'achat, mais aussi à d'autres acteurs. Ce résultat offre la possibilité d'une communication multi-destinataires pour les entreprises qui fournissent des services innovants. Le centre d'achat, l'organisation dans son ensemble ainsi que les clients de l'organisation sont concernés par la communication mise en place par le fournisseur de service. Cette préconisation contribue à faciliter l'adoption et la diffusion des innovations de services, qui sont autant de sources de rupture aux yeux des différents destinataires. 
Malgré les apports de ce travail, plusieurs limites, représentant dans le même temps des pistes d'investigations futures, peuvent être précisées.

Les services abordés au cours des entretiens ne reflètent pas l'ensemble des catégories possibles de services du fait de la taille et des caractéristiques de l'échantillon (majorité de TPE et PME, ou issues du secteur des services). A l'instar de la valeur éthique, d'autres dimensions de la valeur sont ainsi susceptibles d'exister. II serait donc intéressant de répliquer cette étude en élargissant cet échantillon à d'autres types d'entreprises.

D'autre part, pour vérifier l'existence et apprécier l'importance des différentes sources de valeur identifiées dans cette recherche, une étude quantitative serait particulièrement intéressante à mener. Elle permettrait d'approfondir l'ensemble des résultats obtenus lors de ce travail.

Cette étude permet donc une meilleure compréhension de la place de la valeur d'une innovation de service dans le développement d'une stratégie de rupture pour les organisations. La modification des règles du jeu concurrentiel peut ainsi se traduire par une proposition alternative de valeur axée sur des dimensions non-utilitaires. Cette évolution entraîne nécessairement des modifications au niveau de certaines composantes de la chaîne de valeur, modifications qu'il est nécessaire de prendre en compte afin d'assurer le succès des stratégies de rupture.

\section{Bibliographie}

ASSElineAU, A., PIRE-LeChAlARD, P., 2009, "Le développement durable: une voie de rupture stratégique ?", Revue Management et Avenir, 6, 26, pp. 280-299.

AZIMONT, F., COVA, B., SALLE, R., 1999, "Vente de solutions et marketing de projets : une même recherche d'intimité client pour une construction conjointe de l'offre et la demande", Revue Française du Marketing, 173-174, pp. 131-140. 
CORSARO, D., SNEHOTA, I., 2010, "Searching for relationship value in Business Markets: are we missing something?", Industrial Marketing Management, 39, 6, pp 986-995.

COVA, B., SALLE, R., 1992, "L'évolution de la modélisation du comportement d'achat industriel : panorama des nouveaux courants de recherche", Recherche et Applications en Marketing, 7, 2, pp. 83-106.

DE BRENTANI, U., 1995, "New industrial service development: scenarios for success and failure", Journal of Business Research, 32, 2, pp. 93-103.

DE BRENTANI, U., RAGOT, E., 1996, “Developing new business-to-business professional services: what factors impact performance?", Industrial Marketing Management, 25, 517530.

DROEGE, H., HILDEBRAND, D., FORCADA, M. A., 2009, "Innovation in services: present findings, and future pathways", Journal of Service Management, 20, 2, pp. 131-155.

FILSER, M., PLICHON, V., 2004, "La valeur du comportement de magasinage: statut théorique et apports au positionnement de l'enseigne", Revue Française de Gestion, 30, 148, pp. 29-43.

FLINT, D. J., WOODRUFF, R. B., GARDIAL, S. F., 2002, "Exploring the phenomenon of customers' desired value change in a Business-to-Business context", Journal of Marketing, 66, 4, pp. 102-117.

GALLOUJ, F., WEINSTEIN, O., 1997, "Innovation in services", Research Policy, 26, 4-5, p. 537556.

GALLOUJ, F., SAVONA, M., 2009, "Innovation in services: a review of the debate and a research agenda", Journal of Evolutionary Economics, 19, 2, pp. 149-172.

GRÖNROOS C., 2011, "A service perspective on business relationships: the value creation, interaction and marketing interface", Industrial Marketing Management, 40, 2, pp. 240-247.

HAMEL, G. (1998a), "The challenge today: changing the rules of the game", Business Strategy Review, 9, 2, pp. 19-26.

HAMEL, G. (1998b), "Strategy innovation and the quest for value", Sloan Management Review, 39, 2, pp. 7-14.

HOLBROOK, M. B., 1994, "The nature of customer value: an axiology of services in the consumption experience", in RUST, R. et OLIVER, R. L., "Service quality: new directions in theory and practice", Sage Publications, Thousand Oaks, pp. 21-71.

HOLBROOK, M. B., 1999, "Introduction to consumer value”, in HOLBROOK, M. B., "Consumer value: a framework for analysis and research", Routledge, New-York, pp. 1-28. 
HOLBROOK, M. B., 2006, "Consumption experience, customer value, and subjective personal introspection: an illustrative photographic essay", Journal of Business Research, 59, 6, pp. 714-725.

LA, V., PATTERSON, P., STYLES, C., 2009, "Client-perceived performance and value in professional B2B services: an international perspective", Journal of International Business Studies, 40, 2, pp. 274-300.

LE NAGARD-ASSYAG, E., MANCEAU, D., 2005, Marketing des nouveaux produits: de la création au lancement, Dunod, Paris.

LEHMANN-ORTEGA, L., ROY, P., 2009, "États des lieux : les stratégies de rupture" Revue Française de Gestion, 35, 197, pp.113-126.

LEHMANN-ORTEGA, L., SCHOETTL J-M., 2005, "Rupture et perturbation : les deux formes de l'innovation stratégique", Atelier de l'Association Internationale de Management Stratégique sur les stratégies de rupture, Lille, actes électroniques.

LINDGREEN, A., WYNSTRA, F., 2005, "Value in business markets: what do we know? Where are we going?", Industrial Marketing Management, 34, 7, pp. 732-748.

LOVELOCK, C., WIRTZ, J., LAPERT, D., 2004, Marketing des services, 5 ème édition, Pearson Education, Paris.

MALAVAL, P., BENAROYA, C., 2009, Marketing Business to Business, $4{ }^{\text {ème }}$ édition, Pearson Education, Paris.

MARKIDES, C. (1997), "Strategic Innovation", Sloan Management Review, 38, 3, pp. 9-23.

MARKIDES, C. (1998), "Strategic Innovation in established companies", Sloan Management Review, 39, 3, pp. 31-42.

MARKIDES, C. (1999), "A dynamic view of strategy", Sloan Management Review, 40, 3, pp. 55-63.

MENCARELLI, R., 2008, "L'interaction lieu - objet comme conceptualisation de l'expérience vécue : test d'un modèle intégrateur", Recherche et Applications en Marketing, 23, 3, pp. 5169.

METAIS, E., DAUCHY, D., HOURQUET, P. G., 2009, “Les leviers de la déconstruction stratégique : le cas Logan", 197, pp. 147-162

PACHE G. ET DES GARETS V., 1997, Les relations interorganisationnelles dans les canaux de distribution : les dimensions logistiques, Recherche et Applications en Marketing, 12, 2, pp. 61-82. 
PINARD-LEGRY, J., MARION, F., SALLE, R., 1996, Mener des études de satisfaction clients en milieu Business to Business, Décisions Marketing, 8, 83-91.

PHAN, M., 2007, "Innovation de services : étude de cas du Plaza Athénée Paris", Décisions Marketing, 48, octobre-décembre 2007, pp. 9-19.

RIVIERE, A., 2009, "Les effets des stratégies d'enrichissement de produits sur la valeur perçue d'un bien complexe. Une application au secteur automobile", Doctorat de Sciences de Gestion, Université François Rabelais de Tours.

ULAGA, W., CHACOUR, S., 2001, "Measuring customer-perceived value in business markets", Industrial Marketing Management, 30, 6, pp. 525-540.

VARGO, S. L., LUSCH, R. F., 2004, "Evolving to a new dominant logic for marketing", Journal of Marketing, 68, 1, pp. 1-17.

ZEITHAML, V. A., 1988, "Consumer perceptions of price, quality, and value: a means-end model and synthesis of evidence", Journal of Marketing, 52, 3, pp. 2-22. 